\title{
Comparison of the enzymatic and explant methods for the culture of keratinocytes isolated from human foreskin
}

\author{
MAHMOUD ORAZIZADEH ${ }^{1,2}$, MAHMOUD HASHEMITABAR $^{1,2}$, SOMAYEH BAHRAMZADEH $^{2}$ \\ FRESHTEH NEJAD DEHBASHI ${ }^{1}$ and SADEGH SAREMY ${ }^{1}$ \\ ${ }^{1}$ Department of Anatomical Sciences, Faculty of Medicine, ${ }^{2}$ Cellular and Molecular Research Center, \\ Ahvaz Jundishapur University of Medical Sciences, Ahvaz, Khuzestan 61357-15794, Iran
}

Received January 20, 2015; Accepted January 30, 2015

DOI: $10.3892 /$ br.2015.442

\begin{abstract}
Currently, culture and growth keratinocytes are important stages in achieving a reliable and reproducible skin tissue. In the present study, two different methods, enzymatic and explant methods, for keratinocytes isolation from human foreskin were compared. Foreskins were cut into $2-3 \mathrm{~mm}$ pieces and placed in trypsin at $4{ }^{\circ} \mathrm{C}$ overnight for separation of the epidermis from the dermis. Subsequently, these samples were divided into two groups: i) Keratinocytes separated from the epidermis by trypsin and ii) by the explant method. These keratinocytes were divided into two groups: i) With no feeder layer and ii) onto a type I collagen scaffold. The cells were evaluated using immunocytochemistry and 4',6-diamidine-2'-phenylindole dihydrochloride (DAPI) staining. In the enzymatic treatment, after 7-10 days no attached cells were found in the cell culture dishes. In the explant method, keratinocytes were separated after $\sim 24 \mathrm{~h}$, attached rapidly and formed big colonies into a collagen scaffold. In the absence of a feeder layer, small colonies were developed with rapid loss of proliferation within 2-3 days. Keratinocytes showed positive immunoreactivity for the pan-cytokeratin marker and keratinocytes' nuclei were clearly observed. This method could be applied and developed as a component of skin substitutes to treat burns and wounds and also in laboratory testing.
\end{abstract}

\section{Introduction}

The skin is the largest organ in the body that is divided into two anatomically distinct regions, the dermis and epidermis. The normal structure and function of this organ is dependent on the intact epidermis anchored to its vascular, elastic dermis $(1,2)$. Fibroblasts are the most prevalent cell types in

Correspondence to: Dr Somayeh Bahramzadeh, Cellular and Molecular Research Center, Ahvaz Jundishapur University of Medical Sciences, Ahvaz, Khuzestan 61357-15794, Iran

E-mail: bahramzade2007@gmail.com

Key words: keratinocytes, human foreskin, enzymatic method, explant method the dermis, which produce different growth factors that induce proliferation of keratinocytes in vivo and in vitro (3). The principal cell type of the epidermis is the keratinocyte $(1,4)$, which is a small epithelial cell, located at the top of the epidermal basal membrane and characterized by a low division rate $(5,6)$.

Thus far, various enzymatic methods for dermal-epidermal separation have been applied (6-8). For instance, trypsin separates suprabasal hemidesmosomes that causes the basal layer cells to attach to the dermal layer (6). Thermolysin is another enzyme that selectively separates desmosomes $(5,9)$ and is able to separate the epidermis at the basal membrane zone level $(5,10)$.

Although the dissociation method of keratinocytes in primary culture is well-established, attempts to acquire purified adult stem-cell like/progenitor keratinocytes from whole human skin are still ongoing. In particular, different techniques are currently being applied to achieve high purity or homogeneous primary cultures enriched in keratinocyte progenitor/precursor cells. These include filtration, density gradient centrifugation and fluorescence-activated cell sorting using cell surface antibodies, as well as differential adhesion to enrich the cells that rapidly attach to particular substrates (11).

A previous study showed that when keratinocytes/precursor cells separate from the epidermal layer using enzymatic treatment, a number of these cells will not be able to develop colonies, due to their state of differentiation. Only 3-4\% of the keratinocytes can form colonies, even under optimal culture conditions. The formation and confluence of the colonies are directly associated with the keratinocyte layer produced, which will be used as a graft (9).

Numerous studies have been performed on the foreskin of children, whose cells have an extremely different replicative profile to keratinocytes in comparison to older skin $(1,12,13)$. Previous studies have shown that the keratinocytes appear to migrate out from whole skin explants over the first few days in culture (14). Additionally, certain studies have shown that these early migrating cells originate from the basal layer of the epidermis, and fibroblasts do not grow out from adult human skin explants until several days after the appearance of keratinocytes $(14,15)$. This time lag between the migration of keratinocytes versus fibroblasts can be used to reach the pure keratinocytes. Explant-derived keratinocytes can be grown rapidly to multiple passages using the current methods 
of culture, and notably, the original explants can be recycled and used as a continuing source of keratinocytes. Using previous data associated with the explants culture of children's foreskin, the present study aimed to establish a feasible method in the separation and growth of differentiating keratinocytes in culture medium.

\section{Materials and methods}

Tissue collection. Immediately following circumcision of children (age range, 8 day-2 year old), and obtaining parental consent, the foreskin samples were collected from a private clinic and were kept in the bottle containing transfer medium on ice during transportation. The transfer medium was phosphate-buffered saline (PBS) containing $0.5 \mu \mathrm{g} / \mathrm{ml}$ amphotericin $\mathrm{B}, 100 \mathrm{IU} / \mathrm{ml}$ gentamycin, $100 \mathrm{IU} / \mathrm{ml}$ penicillin and $100 \mu \mathrm{g} / \mathrm{ml}$ streptomycine (all from Gibco Life Technologies, Grand Island, NY, USA). In total, 20 samples were collected during 2012.

Tissue preparation. Method I: The procedure was started immediately in the cell culture room of the Cellular and Molecular Research Center (CMRC; Ahvaz, Iran). The samples were placed in $70 \%$ ethanol for $10 \mathrm{sec}$ and washed 3-5 times with PBS. The whole hypodermis layer and associated blood vessels were discarded. Subsequently, the samples were cut into $2-3 \mathrm{~mm}$ pieces and put in $0.25 \%$ trypsin (Gibco Life Technologies) at $4^{\circ} \mathrm{C}$ overnight. During the overnight treatment, the epidermal layer was separated from the dermis. The isolated epidermal layer was placed in a petri dish containing trypsin at $37^{\circ} \mathrm{C}$ for $15 \mathrm{~min}$ until the epidermis became loose and the keratinocytes were released easily. Subsequently, the suspended cells were centrifuged at 1,400 x g for $15 \mathrm{~min}$ and the cell pellet was placed in a $25 \mathrm{~cm}^{2}$ flask and divided into two groups: i) With no feeder layer and ii) onto a type I collagen scaffold (Sigma-Aldrich, St. Louis, MO, USA).

Method II: When the dissociation of the epidermis from the dermis was performed using trypsin, explants of isolated epidermis layer were prepared. The size of the explant pieces were 2-3 mm and 10-15 pieces were placed in each flask, which were divided into two groups: i) With no feeder layer and ii) onto a type I collagen scaffold. One drop full of medium was added to each piece of explant. The medium components contained DMEM/F12, 10\% fetal bovine serum, $100 \mathrm{IU} / \mathrm{ml}$ penicillin, $100 \mu \mathrm{g} / \mathrm{ml}$ streptomycin, $25 \mu \mathrm{g} / \mathrm{ml}$ gentamycin, $2 \mathrm{mM}$ L-glutamine, $1 \mu \mathrm{g} / \mathrm{ml}$ amphotericin $\mathrm{B}, 0.4 \%$ bovine pituitary extract, $0.125 \mathrm{ng} / \mathrm{ml}$ epidermal growth factor human recombinant, $5 \mu \mathrm{g} / \mathrm{ml}$ human insulin, $0.33 \mu \mathrm{g} / \mathrm{ml}$ hydrocortisone, $10 \mu \mathrm{g} / \mathrm{ml}$ human transferrin, $0.39 \mu \mathrm{g} / \mathrm{ml}$ epinephrine and $0.15 \mathrm{mM} \mathrm{CaCl}_{2}$. After one day, $1 \mathrm{ml}$ of medium was added to each flask and the cells began to separate from the explants. During the 4th day, the sections of the non-digested explants were removed and $1 \mathrm{ml}$ of medium was added to the flask. Subsequently, the cells in the flasks were maintained in this condition until the 6th day. During the 6th day, the whole medium was removed and replaced by fresh medium. Cells were checked daily to observe the normal growth.

Immunocytochemistry (ICC). For characterization of the keratinocytes, cultured cells with $50 \%$ confluency were fixed in $2 \%$ paraformaldehyde and permeabilized by $0.1 \%$ Triton $\mathrm{X}-100$ in PBS. Following three washes in PBS, the cells were incubated in a common type of monoclonal antibody, anti-human anti-pan cytokeratin $(0.001 \mu \mathrm{g} / \mathrm{ml})$, produced by the C11 hybrid cells (C11, sc-8018; Santa Cruz Biotechnology, Inc., Dallas, TX, USA) at $4^{\circ} \mathrm{C}$ overnight. After washing three times with PBS, anti-mouse-immunoglobulin-fluorescein isothiocyanate (cat. no. sc-2010; Santa Cruz Biotechnology) was used as a secondary conjugated antibody (16).

The cultures were stained with 4',6-diamidine-2'-phenylindole dihydrochloride (DAPI; blue fluorescence) for $30 \mathrm{~min}$ to show the cell nuclei of the keratinocytes (17). Subsequently, the cells were washed with PBS three times and the positive cells were detected using a fluorescence microscope.

\section{Results}

Enzymatic method. In the enzymatic method, trypsin was applied for separation of the keratinocytes from the epidermal layer, and after $\sim 70$ days no attached cells were found in the two cell culture dishes.

Keratinocyte outgrowth from skin explants. Keratinocytes were separated from the tissue explants after $\sim 24 \mathrm{~h}$. However, to prevent the appearance of fibroblasts at $\sim 5$ days, the epidermal cells were removed after 4 days as an alternative method to obtain keratinocyte primary cultures free of fibroblasts. In the first few days (1-2 days), an early migration phase of keratinocytes from explants were observed and the population of keratinocytes increased due to keratinocyte migration and proliferation.

For generating organotypic co-cultures, epidermal keratinocytes were plated onto the upper surface of the collagen scaffold, where they attached rapidly and formed big colonies. In the absence of a feeder layer, small colonies developed with a rapid loss of proliferation within 2-3 days.

In the collagen-coated flask, keratinocytes were first observed growing out from the explants in a continuous sheet between 24 and $36 \mathrm{~h}$. By day 2, the outgrowth resembled a ring surrounding the explants (Fig. 1A) and during days 3 and 4, this outgrowth continued to expand (Fig. 1B). During day 6 , colonies of cells started to form (Fig. 1C) and on day 12, large colonies of keratinocytes appeared (Fig. 1D).

ICC. The specific marker that cells express as they grow out from the explants was examined. When the cells reached $50 \%$ confluence, dual staining was carried out. Cells were immunostained for pan-cytokeratin, which is a known standard marker for keratinocytes. The keratinocytes population showed strong positive immunoreactivity for the pan-cytokeratin marker (Fig. 2A).

Extremely clear blue staining of the keratinocyte nuclei were observed when the growing cells were stained with the DAPI staining method (Fig. 2B).

\section{Discussion}

In the present study, an explant method was developed for the primary keratinocyte precursor cell culture derived from the foreskin of children. The method was rapid, simple and 


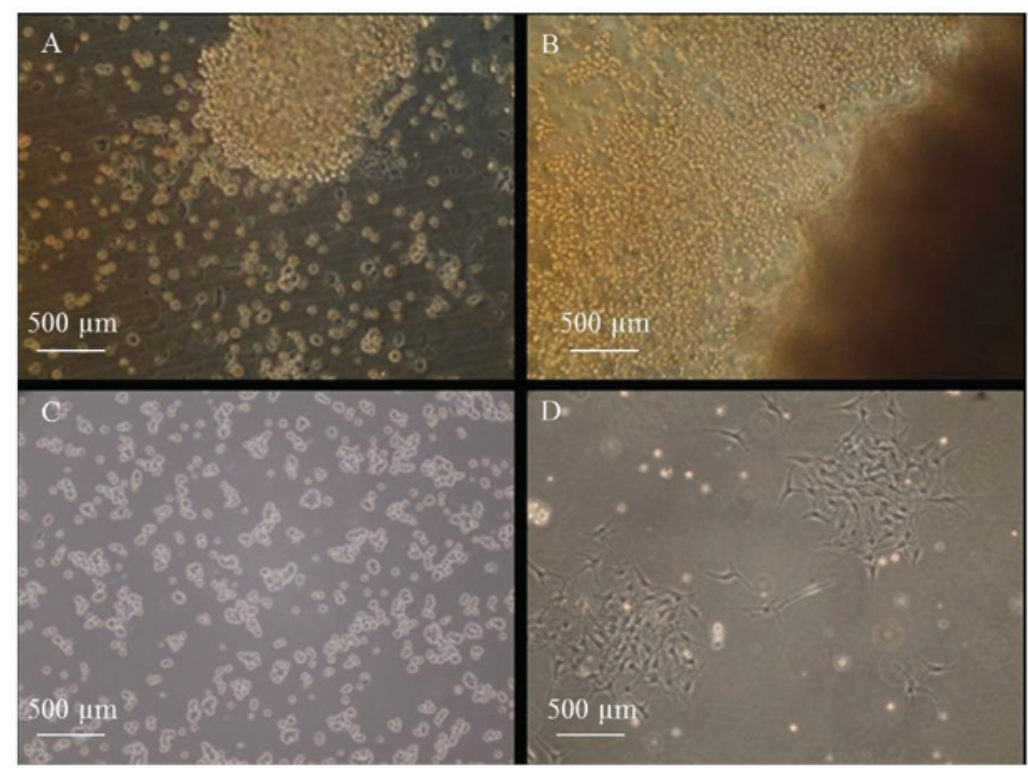

Figure 1. Isolation and culture of keratinocytes from skin explants. (A) During day 2, the keratinocytes resembled a ring surrounding the explants and (B) during days 3 and 4, the keratinocytes continued to expand. (C) During day 6, the keratinocyte colonies started to form and (D) on day 12, big colonies of keratinocytes appeared.
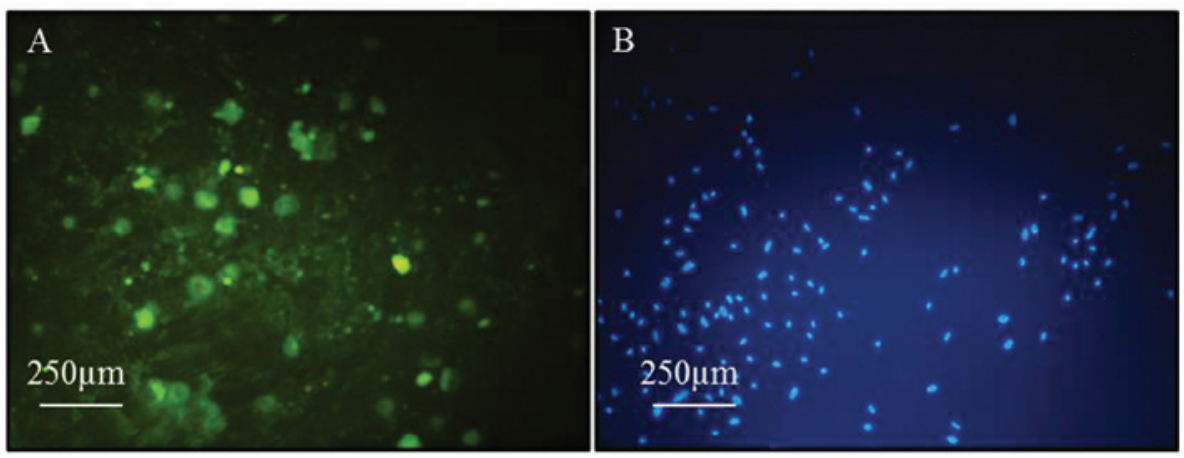

Figure 2. Immunostaining of the keratinocyte culture cells for (A) pan-cytokeratin and (B) nuclei staining by DAPI. DAPI, 4',6-diamidine-2'-phenylindole dihydrochloride.

reliable for the generation and differentiation of keratinocytes without fibroblast contamination. Using this method, the cells showed clear growth characteristics and a typical cell profile presentation.

Two different methods were compared for the separation of keratinocytes from the epidermal layer: Enzymatic method using trypsin and the explant method. In the enzymatic treatment after $\sim 70$ days, no attached cells were found in both cell culture dishes, but using the explant method, the cells showed clear growth characteristics and typical cell profile presentation. Consistent with the present study, Leigh et al (9) showed that when keratinocytes are separated from the epidermal layer by the enzymatic method, even under excellent culture conditions, only 3-4\% of the keratinocytes form colonies. Guo et al (13) investigated the explant method for keratinocyte culture using a serum-free medium known as Epilife ${ }^{\mathrm{TM}}$. The study showed that serial outgrowths of keratinocytes could be harvested from the same explant, which further enhances the utility of this method as it provides the possibility of obtaining much larger numbers of keratinocytes from a single source. Regardless of the present study, McHeik et al (18) isolated keratinocytes following a double-enzymatic digestion (dispase and trypsin) and harvested on average of 11.5 million cells from $4 \mathrm{~cm}^{2}$ of foreskin tissue.

The explant culture method is where a small piece of skin will settle on a culture dish and produce a sizeable outgrowth of cells.. This method has long been employed as a model of wound healing or adult skin epidermal outgrowth rather than a source of keratinocytes for clinical or experimental purposes (19-24). The major limitation of the explant method is due to the fact that fibroblasts also grow out from the same explants and will eventually outgrow the keratinocytes. In the present study, keratinocytes were separated from explants after $\sim 24 \mathrm{~h}$, which is consistent with the previous study by Guo et al (13). As no fibroblast outgrowth was observed until at least 5 days, it was possible to obtain fibroblast-free populations of keratinocytes by removing the explants from the dish after 4 days.

In the present study, in the first few days (1-2 days) an early migration phase of keratinocytes from explants was observed and the population of keratinocytes increased due to 
keratinocyte migration and proliferation. Consistent with this, the studies by Stoll et al (21) and Clark (25) explained that epidermal reepithelialization in skin wounds can be divided into two phases: i) Early migration lasting 1-2 days during which keratinocytes do not proliferate but migrate rapidly in order to close the wound, and ii) a second phase, beginning at day 3 , which is characterized by strong keratinocyte proliferation. A similar process was observed in explant cultures of human skin.

For generating organotypic co-cultures, epidermal keratinocytes were plated onto the upper surface of the collagen scaffold, where they rapidly attached and formed large colonies. In the absence of a feeder layer, small colonies were developed with a rapid loss of proliferation within 2-3 days. The co-cultured fibroblasts can be demonstrated to produce growth factors in an in vitro system, which are essential for epidermal morphogenesis, as previously explained by Witte and Kao (3). Pajoum Pajoum et al (1) showed that the growth of isolated human skin keratinocytes in modified medium was increased compared to serum-free medium. The different evaluations of the collagen-chitosan scaffold showed that it is important for keratinocyte growth and has a good flexibility in the manipulation of living skin equivalents.

There are numerous studies that have focused on the development of nutritionally optimized, readily defined, reproducible media and culture conditions for keratinocyte cells (26-29). The aims of the present study were not to establish a new medium, and therefore, the Rheinwald and Green (30) protocol was applied. The present results showed that a new combination worked well. The latter protocol utilized DMEM supplemented with growth factors that are mentioned in the present study.

Although Coolen et al (29) showed that keratinocytes can be cultured without a fibroblast feeder layer and fetal calf serum, the present results demonstrated that in the modified medium and onto a type I collagen scaffold (as a feeder layer), keratinocyte cell growth was greater than the condition without a feeder layer, and this is consistent with certain previous studies by Gingras et al (31) and Arpornmaeklong et al (32) in 2007. However, a long-term follow-up study is required to more precisely evaluate its fate following proper grafting.

In conclusion, the keratinocytes obtained from the explant culture were shown to exhibit a specific marker and grow for multiple passages when plated onto the upper surface of the collagen scaffold. From a clinical and practical standpoint, this method provides a useful and simple method for growing large numbers of keratinocytes from only a small biopsy quickly.

Finally, this practical method would be useful for separation and growth of the keratinocyte precursor cell, which is a suitable choice for tissue engineering and cell therapy in a number of skin diseases.

\section{Acknowledgements}

Thepresent study wasfinancially supportedby AhvazJundishapur University of Medical Sciences (grant no. CMRC-60), and experimental studies were performed at CMRC. The authors wish to thank Dr Amir Homayon Zandvakili for the preparation of the foreskin samples.

\section{References}

1. Pajoum Shariati SR, Shokrgozar MA, Vossoughi M and Eslamifar A: In vitro co-culture of human skin keratinocytes and fibroblasts on a biocompatible and biodegradable scaffold. Iran Biomed J 13: 169-177, 2009.

2. Yannas IV: What criteria should be used for designing artificial skin replacements and how well do the current grafting materials meet these criteria. J Trauma 24 (Suppl 9): S29-S39, 1984.

3. Witte RP and Kao WJ: Keratinocyte-fibroblast paracrine interaction: The effects of substrate and culture condition. Biomaterials 26: 3673-3682, 2005.

4. Parenteau NL, Nolte CM, Bilbo P, Rosenberg M, Wilkins LM, Johnson EW, Watson S, Mason VS and Bell E: Epidermis generated in vitro: Practical considerations and applications. J Cell Biochem 45: 245-251, 1991.

5. Green H: Cultured cells for the treatment of disease. Sci Am 265: 96-102, 1991

6. Gragnani A, Sobral CS and Ferreira LM: Thermolysin in human cultured keratinocyte isolation. Braz J Biol 67: 105-109, 2007.

7. Tompkins RG and Burke JF: Alternative wound coverings. In: Total Burn Care. Herndon D.N (ed). W.B. Saunders Company Ltda, pp 164-172, 1996.

8. Morgan JR and Yarmush M: Bioengineered skin substitutes. Sci Med 4: 6-15, 1997.

9. Leigh IM, Lane EB and Watt FM (eds): The Keratinocyte Handbook. 1st edition. Cambridge University Press, Cambridge, p 566, 1995.

10. Walzer C, Benathan M and Frenk E: Thermolysin treatment: A new method for dermo-epidermal separation. J Invest Dermatol 92: 78-81, 1989.

11. Kaur P and Li A: Adhesive properties of human basal epidermal cells: An analysis of keratinocyte stem cells, transit amplifying cells, and postmitotic differentiating cells. J Invest Dermatol 114: 413-420, 2000

12. Barrandon $\mathrm{Y}$ and Green H: Three clonal types of keratinocyte with different capacities for multiplication. Proc Natl Acad Sci USA 84: 2302-2306, 1987.

13. Guo A and Jahoda CA: An improved method of human keratinocyte culture from skin explants: Cell expansion is linked to markers of activated progenitor cells. Exp Dermatol 18: 720-726, 2009.

14. Taylor JR, Halprin KM, Levine V and Woodyard C: Effects of methotrexate in vitro on epidermal cell proliferation. Br J Dermatol 108: 45-61, 1983.

15. Van Der Schueren B, Cassiman J-J and Van Den Berghe H: Morphological characteristics of epithelial and fibroblastic cells growing out from biopsies of human skin. J Invest Dermatol 74: 29-35, 1980.

16. Chen TA, Halliwell RE and Hill PB: Failure of extracts from Malassezia pachydermatis to stimulate canine keratinocyte proliferation in vitro. Vet Dermatol 13: 323-329, 2002.

17. Fischer TW, Zbytek B, Sayre RM, Apostolov EO, Basnakian AG, Sweatman TW, Wortsman J, Elsner P and Slominski A: Melatonin increases survival of $\mathrm{HaCaT}$ keratinocytes by suppressing UV-induced apoptosis. J Pineal Res 40: 18-26, 2006.

18. McHeik JN, Barrault C, Bernard FX and Levard G: Quantitative and qualitative study in keratinocytes from foreskin in children: Perspective application in paediatric burns. Burns 36: 1277-1282, 2010.

19. Halprin KM, Lueder M and Fusenig NE: Growth and differentiation of postembryonic mouse epidermal cells in explant cultures. J Invest Dermatol 72: 88-98, 1979.

20. Hammar H: Stimulated mouse ear epidermis in explant cultureThe effect of retinoic acid and hexadecane. Arch Dermatol Res 270: 469-481, 1981.

21. Stoll SWK, Kansra S and Elder JT: Keratinocyte outgrowth from human skin explant cultures is dependent upon p38 signaling. Wound Repair Regen 11: 346-353, 2003.

22. Karasek MA: In vitro culture of human skin epithelial cells. J Invest Dermatol 47: 533-540, 1966.

23. Koeper L-M, Schulz A, Ahr HJ and Vohr H-W: In vitro differentiation of skin sensitizers by cell signaling pathways. Toxicology 242: 144-152, 2007.

24. Mutasim DF, Vaughan A, Supapannachart N and Farooqui J: Skin explant culture: A reliable method for detecting pemphigoid antibodies in pemphigoid sera that are negative by standard immunofluorescence and immunoblotting. J Invest Dermatol 101: 624-627, 1993. 
25. Clark R and Henson PM: The Molecular and Cellular Biology of Wound Repair. Springer, Berlin, 1996.

26. Rosdy M and Clauss L: Complete human epidermal cell differentiation in chemically defined medium at the air-liquid interface on inert filter substrates. J Invest Dermatol 95: 409-414, 1990.

27. Barreca A, De Luca M, Del Monte P, Bondanza S, Damonte G, Cariola G, Di Marco E, Giordano G, Cancedda R and Minuto F: In vitro paracrine regulation of human keratinocyte growth by fibroblast-derived insulin-like growth factors. J Cell Physiol 151: 262-268, 1992.

28. Matsumoto K, Hashimoto K, Yoshikawa K and Nakamura T: Marked stimulation of growth and motility of human keratinocytes by hepatocyte growth factor. Exp Cell Res 196: 114-120, 1991.
29. Coolen NA, Verkerk M, Reijnen L, Vlig M, van den Bogaerdt AJ, Breetveld M, Gibbs S, Middelkoop E and Ulrich MM: Culture of keratinocytes for transplantation without the need of feeder layer cells. Cell Transplant 16: 649-661, 2007.

30. Rheinwald JG and Green H: Serial cultivation of strains of human epidermal keratinocytes: The formation of keratinizing colonies from single cells. Cell 6: 331-343, 1975.

31. Gingras M, Paradis I and Berthod F: Nerve regeneration in a collagen-chitosan tissue-engineered skin transplanted on nude mice. Biomaterials 24:1653-1661, 2003.

32. Arpornmaeklong P, Suwatwirote N, Pripatnanont $P$ and Oungbho K: Growth and differentiation of mouse osteoblasts on chitosan-collagen sponges. Int J Oral Maxillofac Surg 36: 328-337, 2007. 